\title{
Real Time Implementation of SIGN LMS Adaptive Filters using Xilinx System Generator
}

\author{
Anand $\mathrm{R}^{\#}$, Veni $\mathrm{S}^{*}$ \\ \# Department, of Electronics and communication Engineering, Sona college of technology, Salem-637502, India \& \\ Research Scholar, Amrita School of Engineering, Coimbatore, Amrita Vishwa Vidyapeetham, Amrita University, \\ India.641112 \\ E-mail: anand.r@sonatech.ac.in
}

* Department, of Electronics and communication Engineering, Amrita Vishwa Vidyapeetham,

Amrita University, Coimbatore, India

E-mail: s_veni@cb.amrita.edu

\begin{abstract}
Sign Least Mean Square (SLMS) adaptive filter can adapt dynamically based on corresponding filter output. One of the major applications of adaptive filter is Noise cancellation. In real time applications like medical computing, speed of the process developing hardware is essential hence the hardware realization of SLMS adaptive filter using Xilinx System generator is proposed in this work. The propose architecture aims to reduce convergence rate, path delay and increasing speed. In this work (i) Modified architecture is designed for a 8-tap SLMS adaptive filter and (ii) multiplier less structure for Modified DLMS Filter. The designed architecture tested for ECG signal. The functionality of the algorithm is verified in MATLAB with various ECG data from the MIT-BIH database as input. Both LMS and SLMS are designed, simulated, synthesized and implemented in Virtex-5 FPGA using Xilnix ISE 14.3 . The result shows $5 \%$ decrease in total real time router completion and also decrease in the number of adders and subtractors, the maximum combinational path delay has been reduced by $48.84 \%$ in Systolic Sign LMS Filter when compared to LMS Filter.
\end{abstract}

Keywords - Adaptive filter, Least mean square(LMS), Sign Least mean square(SLMS) algorithm, systolic architecture.

\section{INTRODUCTION}

Adaptive filters are mainly used in the field such as Digital signal processing such as adaptive equalization, system identification and noise cancellation etc. All these applications are based on the LMS because it has easy calculation and high performance[1]. Thus Roy et.al., developed the Delay Least Mean Square (DLMS) algorithm for the Very Large Scale Integrated(VLSI) design of LMS adaptive digital filter. It is observed from this paper that the path delay and convergence rate problems are present due to the feedback error term. It is needed for updating all the corresponding weights of the adaptive filter. In case, the hardware implementation, It is essential to reduce path delay, area and power and increase the speed of the structure.

Lan-Da Van [2]., developed the efficient tree structure for real time digital signal processor using Systolic Architecture for the DLMS Adaptive Filter. With the advancement in VLSI technology it is possible to perform hardware realization of the adaptive filters by the use of recent FPGA. As per existing work

the following limitations are identified form the real time implementations of adaptive filter (i) Increasing power, delay ,and speed (ii) Increasing cost due to feedback error. With the use of the advanced systolic architecture it is possible to design the hardware realization for SLMS adaptive filter to overcome the above limitations which is addressed in this work.

Muhammad Zia Ur Rahman[3]., developed A couple of easy and effective signal and mistakes nonlinearity filters, which are computationally having free multiplier weight which can be used for noise cancellation in ECG signal. The proposed implementation, the location considerable signal to noise ratios with have less number of computational complexity are needed. These algorithm are usually easy addition, easy shift and it can be achieve exceptional up over the other least mean algorithm (LMS) realizations. Simulation research indicates that the proposed recognition offers higher performance in comparison with present realizations in phrases of SNR and computational complexity.

Muhammad Zia Ur Rahman[4]., advanced, some straightforward and powerful signal and blunder nonlinearity filters because its is having multiplier unfastened weight upgrade circles are utilized for cancelation of commotion in (ECG) indicators. The execution is suitable for packages, as an example, biotelemetry, in which massive SNR with less number of computational multifaceted nature are required. Those plans for the most element utilize fundamental growth, shift and it can be achieve widespread pace up over the other (LMS)- primarily a well known. recreation contemplates demonstrates that the proposed architecture gives higher execution contrasted with present attention as some distance as sign to-commotion percentage and computational multifaceted nature.

Uzzal Biswas[5], proposed (ECG) is a technique for monitoring and measuring the electrical exercises of heart. Each bit of ECG is extremely key for the analysis of various 
heart problems.. In this paper, implement a more extensive study for denoising each sorts of commotion required with original ECG signal., power spectral density (PSD), frequency spectrum and convergence, SNR, Percentage Root Mean Square Difference(PRD) and MSE.

The computational time and delays are more for all this architecture proposed. In this work reduction in computational time and delay is achieves with the proposed architecture.

The remaining section are organized as section (II) deals with review of adaptive filter . Section (III) deals with principle of ECG and related filtering algorithm. Section (iv) Elaborates the proposed architecture for SLMS algorithm and Experimental results are discussed in section (V). Conclusion are deals with section(VI).

\section{REVIEW ADAPTIVE FILTER.}

The adaptive filter is defined as, a filter which itself regulate its switch function according to some optimizing parameter [6]. The filter coefficients are up-to-date at every generation until they converge to long-established signal. Centered on the precise signals bought, it makes an attempt to find the most excellent filter design [7]. In a non stationary atmosphere, the filter is anticipated to track time versions and range its filter coefficients automatically. In a stationary environment, the filter is anticipated to converge to the Wiener filter [8].

The operation of adaptive filter involves two processes:

1) The method of adaptive filtering algorithm is outlined as its produces an output signal which is similar to a input signal.

2) Adaptation system, to regulate the filtering parameters (filter switch operate) to the (probably time varying) environment . Often, the (average) square value of the error signal is used as the optimization criterion.

Three types of adaptive algorithms are,

1) Recursive Least Square (RLS) algorithm,

2) Least Mean Square (LMS) algorithm.

One of the major applications of adaptive filters is Noise cancellation. The Figure.1 shows the noise cancellation system which will subtract Nosie form a received signal in an adaptively controlled method [10]. These will enable the system to increase the signal to noise ratio (SNR). Few examples of noise cancellation circuits are normally required for the application such as telephone circuits and electrocardiograph(ECG) and easily identify QRS complex [9] etc.., So the validations of our proposed work to implement a new architecture for detection of Power Line interference noise.

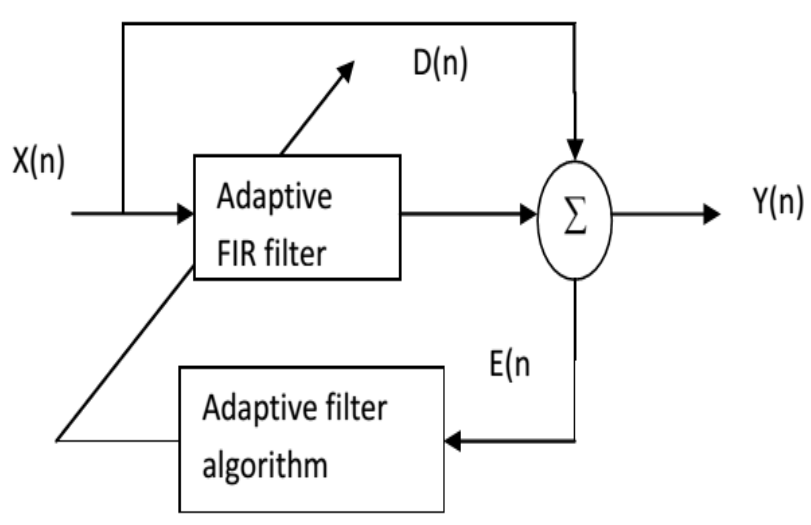

Figure 1: Adaptive Noise cancellation.[5]

The Figure 1 explains General description for the figure are given below, $x(n)$-input signal, d(n)-desired signal, e(n)-error signal, y(n)-filter output.

\section{REVIEW OF PRINCIPLE OF ECG AND RELATED ALGORITHM}

The ECG device detects and amplifies the tiny electrical alterations on the skin that are induced when the heart muscledepolarizes throughout every heartbeat [11]. At leisure, each coronary have a heart muscle ph has a varible charge which is called the membrane, across its cell membrane as shown in figure. 2.

The important of a heartbeat and its incidence time is primary data needed all told varieties of ECG signal process. PQRS signal is employed to spot the events regarding the action, potential, propagation pattern of internal organ signal. The detection of QRS complex is of important significance because of negative detection in overall performance and it will propagate to subsequent method steps and, consequently, restrict the general performance of the machines [15]. Beats that keep undetected represent a a lot of intense errors than do pretend detections; the previous reasonably errors could also be powerful to correct at a later degree inside the chain of process algorithms, while, with a touch of luck, false detections could be removed through, as associate instance, showing class of QRS compelx detector.

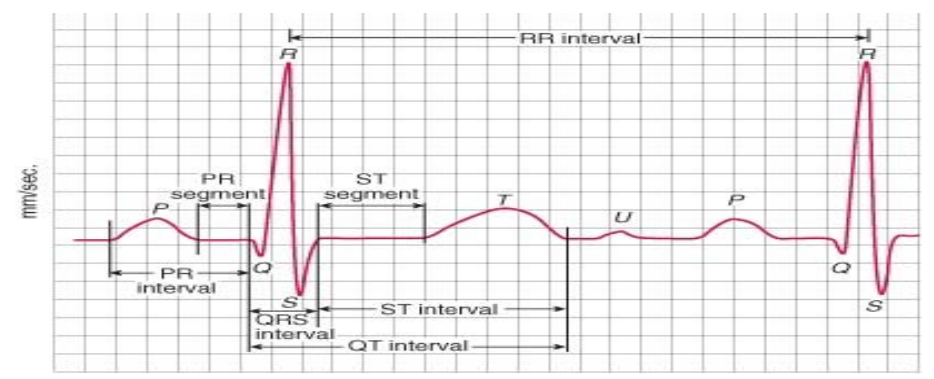

Figure 2: General ECG Signal[11]

A QRS detector [11], should be ready to observe an enormous style of completely different QRS complex morphologies techniques which can be very much useful at the 
graphical record software package of interest. By QRS complex detector [12] we need a good convergence rate and reduce the number of path delays and complexity of structures and also efficiency should better than any other structure. As simulation perspective we are adding Power line interference noise signal to original ECG signal which was collected from MIT-BIH dataset [14] and neglecting that Noise signal form original ECG signal using SIGN LMS adaptive filter.

\section{a. Noises in ECG}

ECG measurements are corrupted with the help of many types of noises. Those of most very important interest area unit Power line interference noise, Baseline wander noise, movement artifacts and Muscle artifacts [21]. This artifacts powerfully influences the ST section, degrades the good signal, frequency changer and produces large amplitude in ECG that can be PQRST waveforms, Each small points which can be very important for scientific pursuit and analysis. Cancellation of those artifacts in electrocardiogram indicators is critical enterprise for higher analysis. During this work transmission line Interference noise is taken into account.

\section{b. Power Line Interference}

- Figure 5 shows the Power Line Interference (PLI) happens through approach of two mechanisms:[14] electrical phenomenon and Inductive coupling. Electrical phenomenon coupling refers back to the transferring of strength among electric circuits through a coupling capacitance and praise among the circuits [24]. The amount of the capacitance is reduces with improving disturbance of the circuits. Inductive coupling always is triggered by coefficient among the conductors

- When electric current transmit via circuits or wires it introduces a flux, in order to induce a modern in corresponding circuits[19].

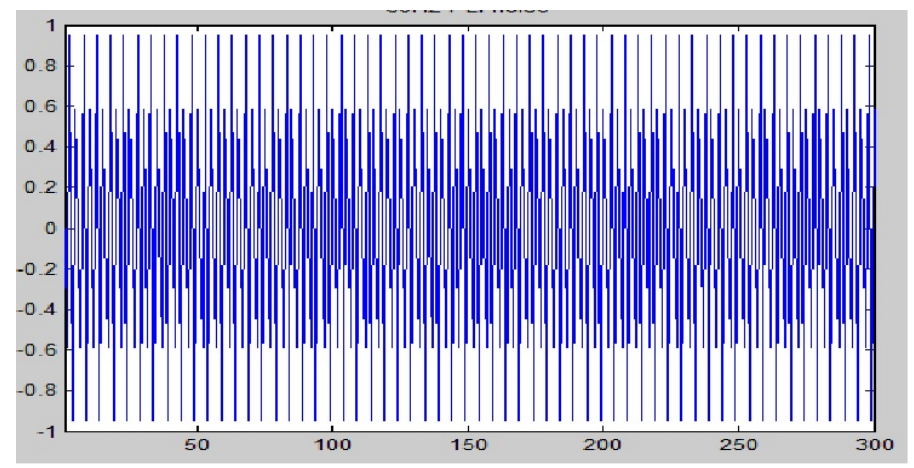

Figure 3: Power Line Interference

On the total, electrical phenomenon coupling is in command of excessive frequency noise at a similar time as inductive coupling produces a less frequency noise because of the mechanism of line interference in ECG signal is to restrict the number of area unit PLI, electrodes ought to be administrated effectively, that there don't seem to be any free wires, and every one accessories have decent protective. The PLI has frequency of both $60 \mathrm{~Hz}$ or $50 \mathrm{~Hz}$ relying at the strength applied.

Fig 4 shows the ECG input signal and PLI noise. The ECG signal is obtained from the database. The ECG signals of 4000 samples is obtained MIT-BIT. This is used as the input for the system.

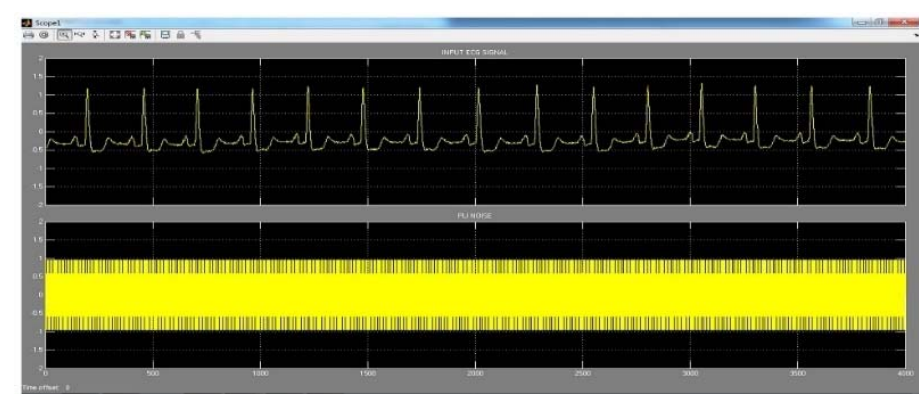

Figure 4: ECG Signal With PLI Noise.

\section{c. LMS Algorithm}

LMS is a one of the applications of adaptive algorithm, it is used to imitate intently a real signal by locating the filter output coefficients that is to introducing the LMS of the error signal i.e. the relation between the desired and the real ECG signal [17]. It is a stochastic gradient descent method in which the weights are varying based on the respective time. LMS algorithm having, the weight update equation is given below. The three basic equations for LMS filter (filter output, Error signal, Estimating weight adaptations).

$$
\begin{gathered}
y(n)=w(n) * x(n) \\
e(n)=d(n)-y(n) \\
w(n+1)=w(n)+2 \mu e(n) x(n)
\end{gathered}
$$

Where,

$\mu$ - The tap Step Size, $\mathrm{w}(\mathrm{n}+1)-$ New weight update for corresponding filter output, $\mathrm{x}(\mathrm{n})$ - The input signal, e(n)- The error signal.

\section{d. Flow Chart For LMS adaptive filter.}

Figure 5 shows the least mean square, the input signal $\mathrm{x}(\mathrm{n})$, and weighted signal $\mathrm{w}(\mathrm{n})$ with initial weight as zero. Convolution performed is done for the various values of $\mathrm{x}(\mathrm{n})$ and $w(n)$. The output of the convolution is subtracted from real signal and noise signal $d(n)$. The minimum error will occur which is moved on to the weighted block and the new weight is updated[16]]. In case if minimum error is zero, the output is obtained directly. 


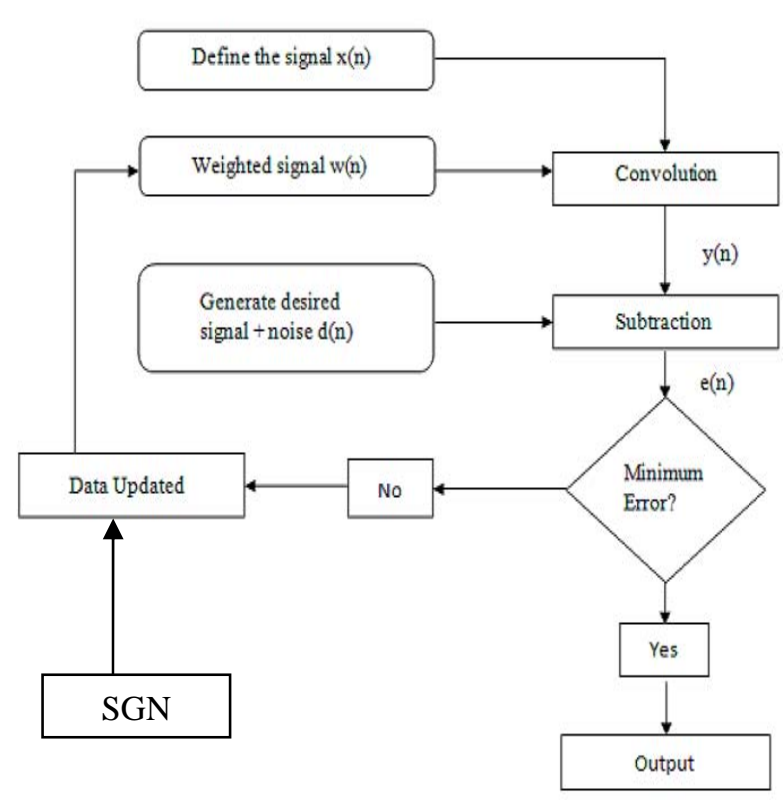

Figure 5: Flow chart for SLMS Adaptive filter

\section{e. Sign LMS Algor,ithm}

Sign based algorithm is mostly adjustive filters area unit computationally advanced with multiplier factor loose weight update loops. These algorithms principally use easy shift and add operations and so achieves extended speed over different least mean sq. based mostly realizations. These algorithms are unit appropriate for applications cherish biotelemetry, wherever massive signal to noise ratios with less machine complexness is needed. Sgn algorithms are dissent from the standard LMS adjustive algorithms by using sgn operate within the weight updating equation. The sgn function as $\mathrm{x}$ can be shown in below equation.

$$
\operatorname{Sgn}(X)=\left(\begin{array}{l}
1: x>0 \\
0: x=0 \\
1: x<0
\end{array}\right)
$$

In this algorithm, the sgn function is applied in the weight update equation to $\mathrm{x}(\mathrm{n})$. This algorithm updating the coefficients of the filter by using the equation is shown in below.

$$
\boldsymbol{w}(\boldsymbol{n}+\mathbf{1})=\boldsymbol{w}(\boldsymbol{n})+2 * \mu * \boldsymbol{S g n}\{\boldsymbol{e}(\boldsymbol{n})\} * \boldsymbol{x}(\boldsymbol{n})
$$

where, Sgn is SIGN conditions.

\section{PROPOSED SYSTOLIC ARCHITECTURE}

In application fields of medical computing, it's essential to resolve linear equations, especially, a really massive-scale linear system of equations are potential. Finding quick, accurate, and price-effective techniques for resolution a very high dimensional linear equation of a system are greatly required for details, every by means of scientists and engineers. Despite the very fact that, we have a tendency to perpetually handling algebra, like LU and SVD decomposition, inversion, and multiplication. Thanks to the prolonged sequences of arithmetic computations, most bigscale algebra is distributed on high-pace digital computers mistreatment properly advanced computer code applications [17]. But a significant disadvantage in process algebra on standard-motive computer systems through computer code program applications is the need for long computation time. Also, during a wide unfold motive pc the most reminiscence isn't massive comfortable to accommodate very big-scale matrices. As a consequence, several long I/O transfers are required additionally to the processor computation time. To alleviate this trouble, mistreatment parallel pc systems has been followed and distinctive reason machines are delivered. The generalized systolic architecture is show in below.

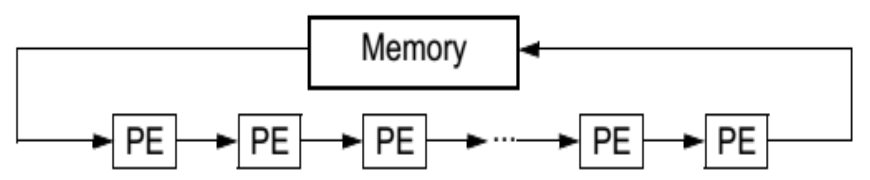

Figure 6:General Systolic architecuture[15]

One choice to the requirement for notably parallel machine electricity is that the association of an enormous vary of same straightforward processors or process parts (PEs). Every PEs has strained personal garage, and is handiest allowed to be relating to neighboring PEs. Consequently all PEs area unit organized during a well-organized form as well as a linear or two-dimensional array. this type of structure, observed as a beat array, provides a good format for VLSI implementation. frequently, interleaved recollections area unit wont to feed statistics into such arrays. Commonly, a beat array contains a rectangular or polygonal shape pure mathematics, but it's viable for it to own any pure mathematics. With VLSI era, it's potential to provide extraordinarily cheaper machine functionality with a system inclusive of a colossal selection of equal little processors ready during a well-structured style. Figure 7 shows a proposed architecture for improving speed of the circuits and adaptation time in receiver is very fast comparing with original architecture. 


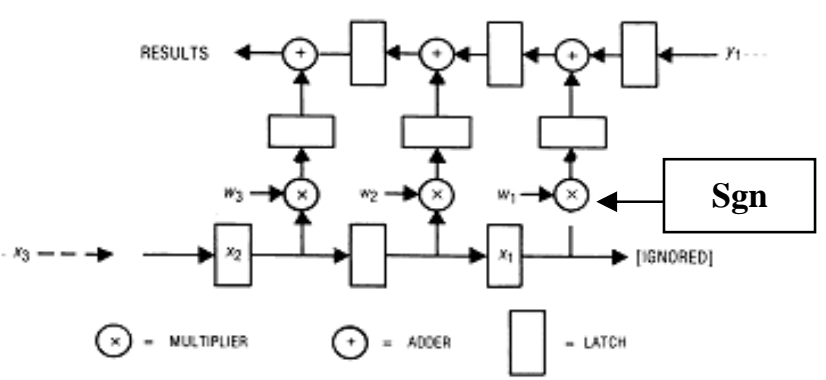

Figure 7:Propoesed Systolic Architecutre[22]

In alternative words, through development in VLSI technology, a affordable array of processors with excessivepace computations is also applied. numerous models of systolic arrays with exclusive information move schemes for matrix operation had been projected. The projected styles are polygon arrays, pipelined arrays, semi broadcast arrays, wave front arrays, and broadcast arrays. In this work, we designed a new architecture which is easily adapt with output and fast computation compared with existing methods.. Figure 7 shows the systolic architecture for FIR filter, which can been designed by using linear mapping technique.

\section{EXPERIMENTAL RESULTS}

\section{a. XILINX SYSTEM GENERATOR MODEL OF 8-TAP LMS FILTER STRUCTURE}

We designed a efficient SIGN LMS adaptive filter for various real time applications. In this we designed a 8-tap LMS filter using equations 6 and 7 .

$$
Y\{n)=\sum_{i=0}^{i=7} w(i, n) x(n-i)
$$

$$
w(i, n+1)=w_{i, n}+2 * \mu * x_{n-i} * e_{n}
$$

Figure 11 indicates four signals (i) input with noise (ii)noise only. (iii) input signal only and (iv) output of the ECG signal. It is observed that the time taken by the output signal is very less in the order of microseconds and also delay is found to be very less compared with the existing architecture. Comparison results in table 1 . The signal strength is better than LMS structure.

As per the equations, where ' $i$ ' is a tap number for the filter and 8 tap adaptive filter is used with tap $=0$ to $\mathrm{i}-1$. Simulation of Systolic 8-tap LMS filter is implemented in Xilinx system generator. Figure 9 shows the output of the systolic 8-tap LMS filter.

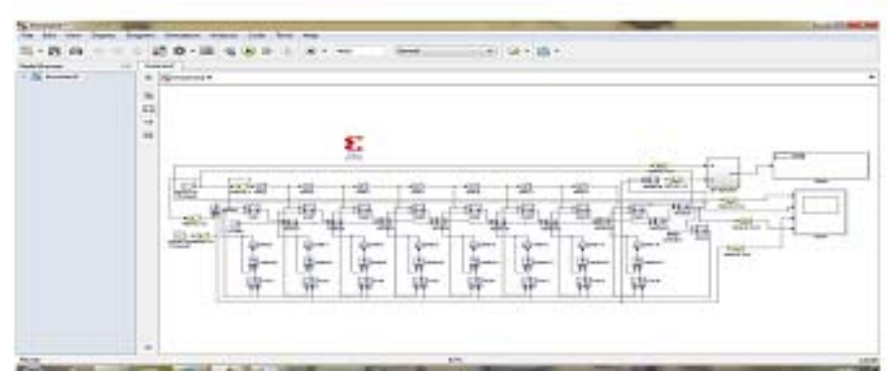

Figure 8: Xilinx System Generator Model of 8-Tap LMS Filter Structure

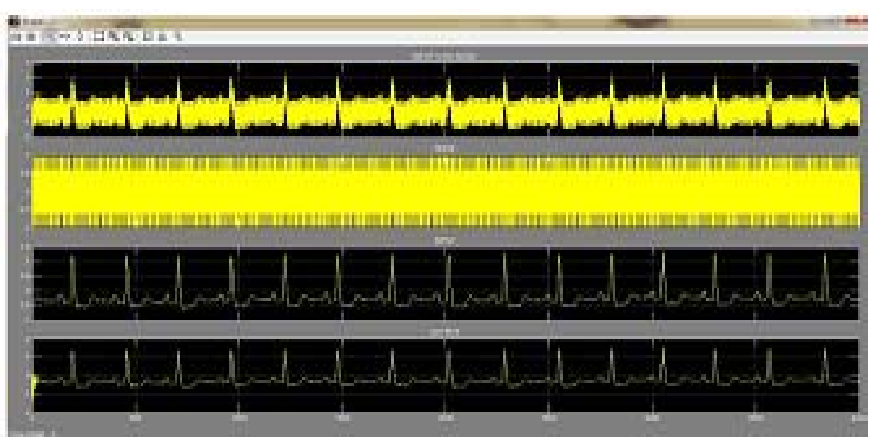

Figure 9: Simulation result of Systolic 8-tap LMS filter in xilinx system generator

Figure 9 indicates four signals (i) input with noise (ii)noise only. (iii) input signal only and (iv) output of the ECG signal. It is observed that the time taken by the output signal is very less in the order of microseconds and also delay is found to be very less. Comparison results in table 1 but signal strength is very low so we go for systolic architecture.

\section{b. XILINX SYSTEM GENERATOR MODEL OF 8-TAP SIGN LMS FILTER STRUCTURE}

We designed a efficient SIGN LMS adaptive filter for various real time applications. In this we designed a 8-tap LMS filter using equations 8 and 9,

$$
Y\{n)=\sum_{i=0}^{i=7} w(i, n) x(n-i)
$$

$$
w(i, n+1)=w_{i, n}+2 * \mu * \operatorname{Sgn}\left(x_{n-i}\right) * e_{n}
$$

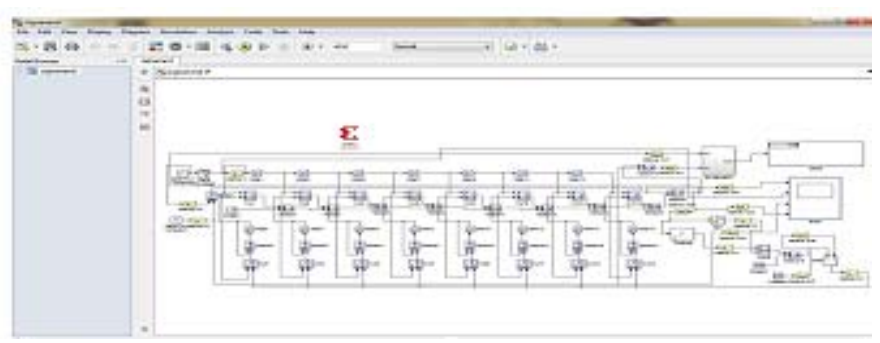


Figure 10: Xilinx System Generator Model of 8-Tap Sign LMS Filter Structure

Figure 10 shows the output of the systolic 8-tap SLMS filter.

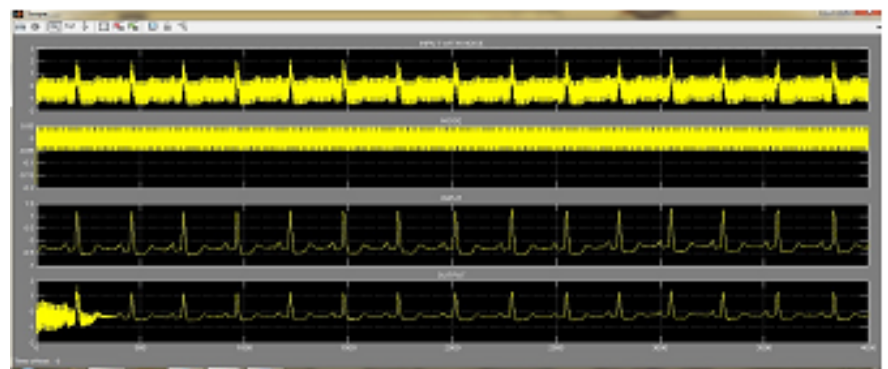

Figure 11: Simulation result of Systolic 8-tap Sign LMS filter in xilinx system generator

\section{c. XILINX SYSTEM GENERATOR MODEL OF 8-TAP} SYSTOLIC LMS FILTER STRUCTURE

The Systolic LMS filter is designed using xilinx System Generator [27] with Electrocardiogram wave as input signal as shown in Figure 10. In this case the noise is power line interference of frequency $60 \mathrm{~Hz}$. Simulation of Systolic 8-tap LMS filter is implemented in xilinx system generator. Figure 10 shows the output of the systolic 8-tap LMS filter.

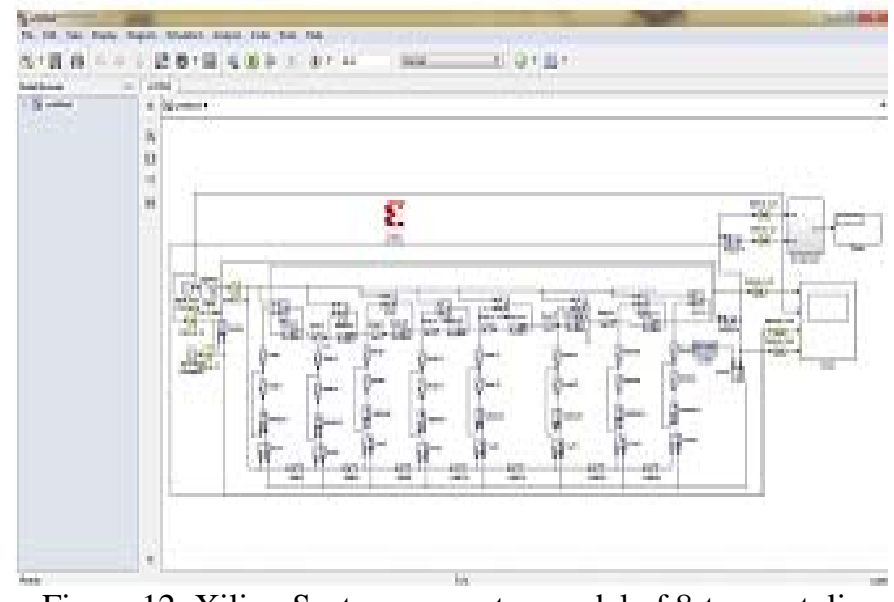

Figure 12: Xilinx System generator model of 8-tap systolic LMS filter structure

Figure 13 indicates four signals (i) input with noise (ii)noise only. (iii) input signal only and (iv) output of the ECG signal. It is observed that the time taken by the output signal is very less in the order of microseconds and also delay is found to be very less compared with the existing architecture. Comparison results in table 1 . The signal strength is better than both LMS and SLMS.

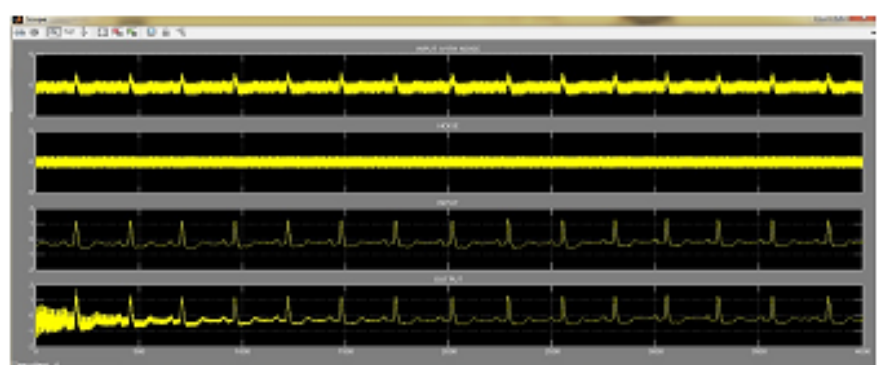

Figure 13: Simulation result of Systolic 8-tap systolic LMS filter in xilinx system generator

\section{d. XILINX SYSTEM GENERATOR MODEL OF 8-TAP SYSTOLIC SLMS FILTER STRUCTURE}

The Systolic Sign LMS filter is designed in Xilinx System Generator with Electrocardiogram wave as input as shown in Figure 10. In this case the noise is power line interference of frequency $60 \mathrm{~Hz}$. Simulation of Systolic 8-tap LMS filter is implemented in xilinx system generator. Figure 14 shows the output of the systolic 8-tap SLMS filter.

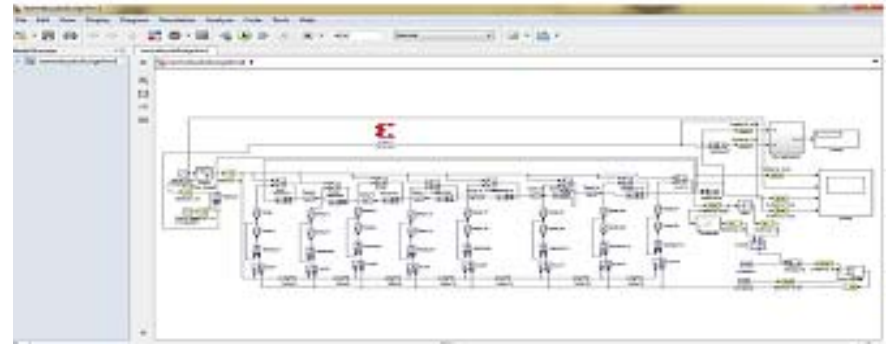

Figure 14: Xilinx system generator model of 8-tap systolic Sign LMS filter structure

Figure 15 indicates four signals (i) input with noise (ii)noise only. (iii) input signal only and (iv) output of the ECG signal. It is observed that the time taken by the output signal is very less in the order of microseconds and also delay is found to be very less compared with the existing architecture [22]. Comparison results in table 1 . The signal strength is better than all existing methods.

From the experimental result we can conclude that there is a improvement in SNR with the proposed in SLMS architecture using systolic array hence the proposed method will be very much suitable for real time implementations of Noise removal of ECG signal[20]. 


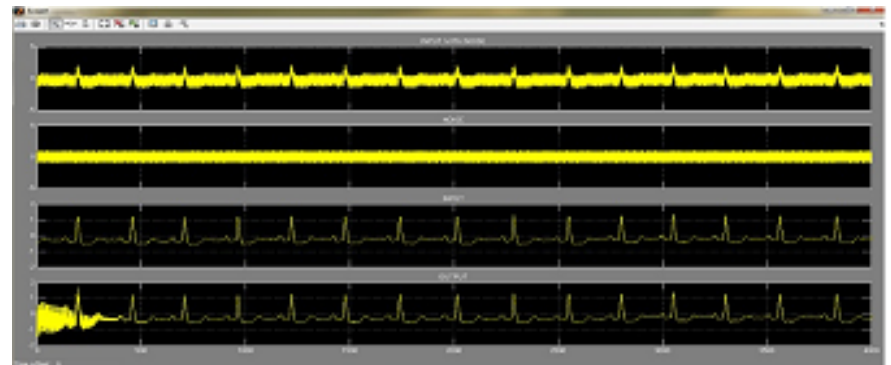

Figure 15: Simulation result of Systolic 8-tap systolic Sign LMS filter in xilinx system generator

\section{e. RESOURCE UTILIZATION OF 8-TAP ADAPTIVE FILTERS}

The resource utilization summary for LMS ,SIGN LMS, SYSTOLIC LMS, SYSTOLIC SIGN LMS is obtained by implementing the above structures in virtex-5. FPGA kit [23].
It is observed from the implementations results listed in Table 2 that maximum combinational path delay has been reduced by $48.84 \%$ in systolic sign LMS filter when compared to LMS filter. From the simulation results the performance of different adaptive filters are analyzed with reference to the SNRs obtained. These filters are then implemented in Virtex -5 kit and the resource utilization of these filters are analyzed. From the table 1 and 2 it is observed that, that the number of LUTS required for adaptive noise cancellation has been reduced by 7.32\% using Systolic Sign LMS filter when compared with the conventional LMS. The result shows $5 \%$ decrease in total real time router completion and also decrease in the number of adders and subtractors. The combinational path delay has been reduced by $48.84 \%$ in Systolic Sign LMS Filter when compared to LMS Filter and also the SNR of the systolic SIGN LMS filter is improved by 13.99\% compared with systolic LMS filter.

Table 1: Comparison of SNR for adaptive filter

\begin{tabular}{|c|c|c|c|c|}
\hline \multirow[t]{2}{*}{ FILTER } & \multicolumn{3}{|c|}{$\begin{array}{c}\text { SNR FOR } \\
\text { RECORD NO. }\end{array}$} & \multirow[t]{2}{*}{ Avg } \\
\hline & 101 & 105 & 228 & \\
\hline LMS & 17.95 & 18.34 & 15.49 & 17.26 \\
\hline BABAMERFAR & 418.84 & SIGN1I8MgS & SYSTP\&B6 & SYSTOLIY7.96 \\
\hline NYYSFOLIC LMS,FH & 25724 & 247.63 & $\begin{array}{l}\text { TMS } \\
46.82 \\
169\end{array}$ & $\begin{array}{r}\text { SHGNLMS } \\
\text { 167.89 } \\
\end{array}$ \\
\hline 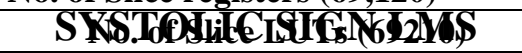 & $836_{76}$ & 894,11 & 88367 & 82814 \\
\hline MaximunFdiefdtiRg frequency & $20.291^{\circ}$ & $91.008^{ \pm 1}$ & $31.445^{5 /}$ & $90.959^{4}$ \\
\hline (MHz) & & & & \\
\hline $\begin{array}{c}\text { Total REAL time to Router } \\
\text { completion(sec) }\end{array}$ & 31 & 21 & 25 & 20 \\
\hline $\begin{array}{l}\text { Total CPU time to Router } \\
\text { completion(sec) }\end{array}$ & 31 & 21 & 25 & 19 \\
\hline No of adders and subtractors & 31 & 35 & 35 & 31 \\
\hline Combinational path delay(ns) & $30.057 \mathrm{~ns}$ & 37.038 & 15.002 & 15.375 \\
\hline No. of IOS & 114 & 226 & 130 & 227 \\
\hline
\end{tabular}

Table 2: Resources utilization of 8-Tap LMS filter Using Vertex -5

\section{CONCLUSIONS}

Least Mean Square algorithm is an adaptive filtering algorithm which aims at the minimization of the sum of the squares of the difference between the desired signal and the filter output. For denoising ECG signal that is found to contain power line interference noise obtained from the MIT-BIH database, different LMS adaptive filters such as 8 - tap LMS, Delay LMS, Modified Delay LMS, SSLMS, SS Delay LMS and SS Modified Delay LMS are designed and simulated using System Generator. From the simulation results the performance of different adaptive filters are analyzed with reference to the SNRs obtained. These filters are then implemented in Virtex -5 kit and the resource utilization of these filters are analyzed. From the analysis it is observed that, that the number of LUTS required for adaptive noise cancellation has been reduced by $7.32 \%$ using Systolic Sign LMS filter when compared with the conventional LMS. The proposed work can be extended for the real-time implementation of ECG signal because of less computational time and reduced delay.

\section{REFERENCES}

[1] Kim, CH-I., Hendrawan Soeleman, and Kaushik Roy. "Ultra-low-power DLMS adaptive filter for hearing aid applications." IEEE Transactions 
on Very Large Scale Integration (VLSI) Systems 11.6 (2003): 10581067.

[2] Lan-Da van, and Wu-Shiung, "An efficient systolic architecture for the DLMS adaptive filter,” in Proc. IEEE Int. Symp. Circuits Syst., May ,2001 pp. 121-124.

[3] Rahman, Muhammad Zia Ur, Rafi Ahamed Shaik, and DV Rama Koti Reddy. "Efficient and simplified adaptive noise cancelers for ECG sensor based remote health monitoring." IEEE Sensors Journal 12.3 (2012): 566-573.

[4] Rahman, Muhammad Zia Ur, Rafi Ahamed Shaik, and DV Rama Koti Reddy. "Efficient sign based normalized adaptive filtering techniques for cancelation of artifacts in ECG signals: Application to wireless biotelemetry." Signal Processing 91.2 (2011): 225-239.

[5] Biswas, Uzzal, et al. "ECG signal denoising by using least-mean-square and normalised-least-mean-square algorithm based adaptive filter." Informatics, Electronics \& Vision (ICIEV), 2014 International Conference on. IEEE, 2014.

[6] J. Arenas-Garc' 1a and A. R. Figueiras-Vidal, "Adaptive combination of proportionate filters for sparse echo cancellation,” Audio, Speech, and Language Processing, IEEE Transactions on, vol. 17, no. 6, pp. 1087-1098, 2009.

[7] B. K. Das and M. Chakraborty, "Sparse adaptive filtering by an adaptive convex combination of the lms and the za-lms algorithms,".

Circuits and Systems I: Regular Papers, IEEE Transactions on, vol. 61, no. 5, pp. 1499-1507, 2014.

[8] S. S. Haykin, Adaptive filter theory. Pearson Education India, 2008.

[9] Laguna P, Jane ' R, Olmos S, Thakor NV, Rix H, Caminal P (1996) Adaptive estimation of QRS complex by the Hermite model for classification and ectopic beat detection. Med Biol Eng Comput 34(1):58-68.

[10] R. L. Das and M. Chakraborty, "Sparse adaptive filters-an overview and some new results," in Circuits and Systems (ISCAS), 2012 IEEE International Symposium on. IEEE, 2012, pp. 2745-2748.

[11] J Khalaf, Ashraf AM, Mostafa M. Ibrahim, and Hesham FA Hamed. "Performance study of adaptive filtering and noise cancellation of artifacts in ECG signals." 2015 17th International Conference on Advanced Communication Technology (ICACT). IEEE, 2015

[12] Satheeskumaran, S., and M. Sabrigiriraj. "A new LMS based noise removal and DWT based R-peak detection in ECG signal for biotelemetry applications." National Academy Science Letters 37.4 (2014): 341-349.

[13] http://www.physionet.org/physiobank/database/mitdb/ MIT-BIH Arrhythmia Database Website. Available [Online]: (viewed at 10.10.2013 at $10.15 \mathrm{PM})$.

[14] M. Maniruzzaman, K. M. S. Billah, U. Biswas, and B. Gain, "Leastmean-square algorithm based adaptive filters for removing power line interference from ECG signal,” in Proc.ICIEV'12, paper 410, pp. 737- 740, May 18-19, 2012.1

[15] C. Torres-Huitzil and M. Arias-Estrada, "Real-time image processing with a compact fpga-based systolic architecture," Real-time imaging, vol. 10, no. 3, pp. 177-187, 2004.

[16] S. Olmos and P. Laguna, "Steady-state MSE convergence analysis in LMS adaptive filters with deterministic reference inputs for biomedical signals," IEEE Trans. Signal Process., vol. 48, no. 8, pp. 2229-2241, Aug. 2000.

[17] L. Biel, O. Pettersson, L. Philipson, and P. Wide, "ECG Analysis: A new approach in human identification," IEEE Trans. Instrum. Meas., vol. 50, no. 3, pp. 808-812, Jun. 2001.

[18] S. Mitra, M. Mitra, and B. B. Chaudhuri, "A rough-set-based inference engine for ECG classification,” IEEE Trans. Instrum. Meas., vol. 55, no. 6, pp. 2198-2206, Dec. 2006.

[19] C. Meyer, J. F. Gavela, and M. Harris, "Combining algorithms in automatic detection of QRS complexes in ECG signals," IEEE Trans. Inf. Tech. Biomed., vol. 10, no. 3, pp. 468-475, Jul. 2006.

[20] Y. Der Lin, Y. Hen Hu, Power-line interference detection and suppression in ECG signal processing, IEEE Transactions on Biomedical Engineering 55 (January) (2008) 354-357.
[21] Sano, Kentaro, Takanori Iizuka, and Satoru Yamamoto. "Systolic architecture for computational fluid dynamics on FPGAs." 15th Annual IEEE Symposium on Field-Programmable Custom Computing Machines (FCCM 2007). IEEE, 2007

[22] Ownby, Matthew, and W. H. Mahmoud. "A design methodology for implementing DSP with Xilinx System Generator for Matlab." SOUTHEASTERN SYMPOSIUM ON SYSTEM THEORY. Vol. 35. 2003.

[23] Rasu, R., P. Shanmuga Sundaram, and N. Santhiyakumari. "FPGA based non-invasive heart rate monitoring system for detecting abnormalities in Fetal." Signal Processing And Communication Engineering Systems (SPACES), 2015 International Conference on. IEEE, 2015. 